\title{
Adenopatía supraclavicular como forma de presentación de un carcinoma de cérvix asociado al complejo esclerosis tuberosa con linfangioleiomiomatosis
}

\author{
Supraclavicular adenopathy presenting as a cervical carcinoma associated with tuberous \\ sclerosis complex and lymphangioleiomyomatosis
}

\author{
Pablo López Mato, Alfonso Varela Fariña, Elena Seco Hernández, Antonio J. Chamorro Fernández \\ Departamento de Medicina Interna, Complexo Hospitalario Universitario de Ourense. SERGAS. Ourense.
}

\begin{abstract}
Resumen
La linfangioleiomiomatosis es una proliferación del tejido muscular broncovascular que recientemente se ha definido como una expresión incompleta de la entidad "complejo esclerosis tuberosa", una facomatosis a la que se asocian diversas neoplasias. Presentamos un caso de carcinoma de cérvix con metástasis supraclaviculares y cervicales, asociado a linfangioleiomiomatosis en el contexto de un "complejo esclerosis tuberosa".

Palabras clave: Adenocarcinoma de cérvix. Linfangioleiomiomatosis. Complejo esclerosis tuberosa. Adenopatía supraclavicular
\end{abstract}

\section{Introducción}

El carcinoma de cérvix uterino supone el 15\% de las neoplasias ginecológicas. Si bien lo más frecuente es la diseminación linfática o por contigüidad a región pelviana y cadenas ilíacas, respectivamente, también están descritas las metástasis a región supraclavicular en un 5-20\% de los pacientes que presentan dicha afectación adenopática locorregional ${ }^{4}$.

La esclerosis tuberosa es una facomatosis de herencia autosómica dominante que se relaciona con mutaciones en los genes supresores de tumores Tuberous Sclerosis Complex 1 y 2 (TSC1 y TSC2), y a nivel clínico se caracteriza por la presencia de hipopigmentaciones cutáneas, angiofibromas faciales, retraso mental y/o tumores benignos de distinto tipo, entre ellos rabdomiomas cardiacos, angiomiolipomas renales y tumores de sistema nervioso central ${ }^{2}$. Recientemente se ha definido la entidad "Complejo Esclerosis Tuberosa" (CET), en la que se incluye la linfangioleiomiomatosis (LAM), una proliferación del músculo liso broncovascular, como parte de la misma o bien una expresión incompleta de dicha entidad, en el caso de sólo estar presente dicha manifestación ${ }^{3}$.

Presentamos un caso de de carcinoma de cérvix con metástasis linfática supraclavicular y cervical, asociado a linfangioleiomiomatosis en el contexto de un "complejo esclerosis tuberosa".

\begin{abstract}
Lymphangioleiomyomatosis is a proliferation of the bronchovascular smooth muscle, which has been defined recently as an incomplete expression of the "Tuberous Sclerosis Complex" entity, a phacomatosis related to several neoplasms. We introduce a case of adenocarcinoma of the cervix with supraclavicular and neck metastases, associated with lymphangioleiomyomatosis in the context of "Tuberous Sclerosis Complex".

Key words. Adenocarcinoma of the cervix. Tuberous sclerosis complex. Lymphangioleiomyomatosis, Supraclavicular adenopathy

\section{Caso clínico}

Mujer de 79 años sin antecedentes médico de interés que consultó por tumoración cervical izquierda no dolorosa de 1 mes de evolución, refiriendo además leve sangrado vaginal en el interrogatorio por aparatos y sistemas. Durante la entrevista se apreciaba un nivel intelectual probablemente por debajo de la media. No existía historia de comicialidad ni otros datos de focalidad neurológica.

En la exploración física destacaban múltiples adenopatías cervicales y supraclaviculares de consistencia no pétrea, de predominio en región cervical izquierda; y una discreta ptosis palpebral con enoftalmos, sin miosis o anhidrosis ipsilaterales. Se apreciaban en región perinasal unas lesiones hiperpigmentadas de unos 2-3 mm de diámetro (Figura 1), sin observarse lesiones similares en ninguna otra zona corporal.
\end{abstract}

Figura 1. Lesiones faciales hiperpigmentadas de predominio perinasal, sugerentes de angiofibromas

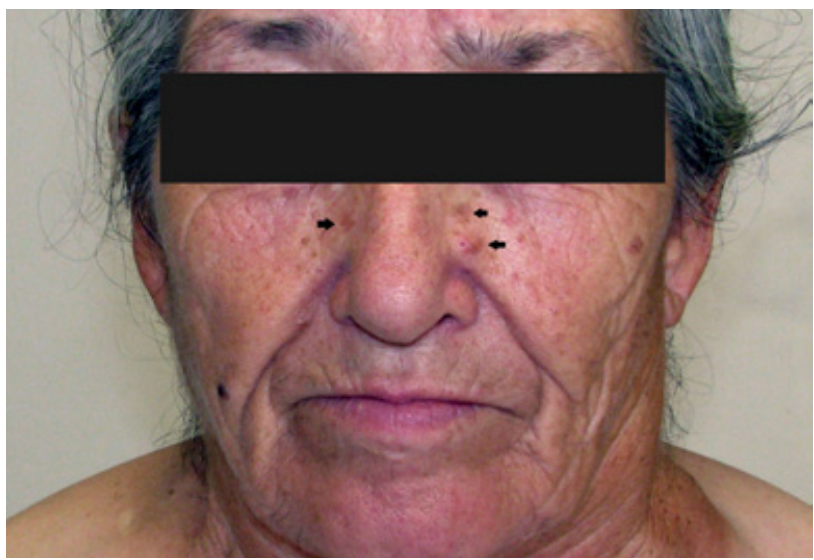


Figura 2. TAC torácico donde se aprecian las imágenes "en panal de abeja" características de linfangioleiomiomatosis

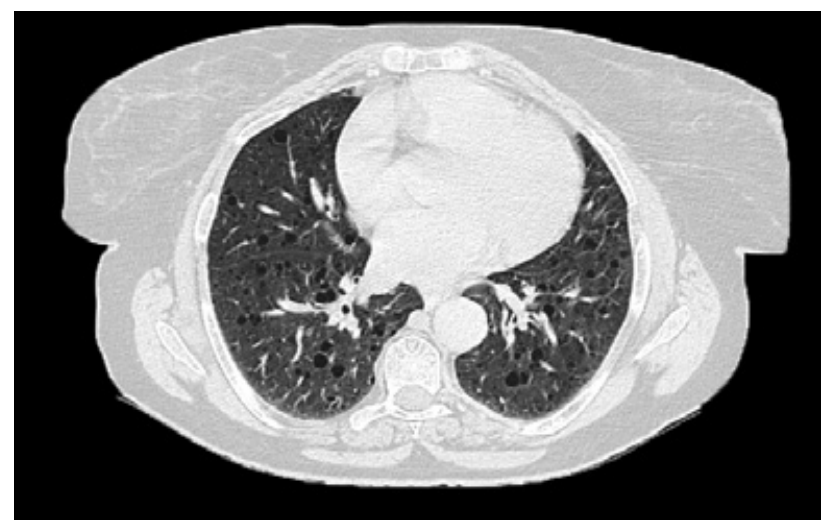

La exploración ginecológica mostró una masa heterogénea con algunas zonas necróticas en tercio superior de la vagina, con un tamaño medido por ecografía de unos 6-7 cm y de probable origen en cérvix o cuerpo uterino.

En la analítica se objetivó una discreta leucocitosis neutrofílica y elevación de la velocidad de sedimentación globular $(44 \mathrm{~mm} /$ 1h), sin otras alteraciones en la bioquímica ni en la coagulación.

La radiografía de tórax mostraba un posible aumento generalizado de la trama intersticial. La ecografía cervical presentaba un conglomerado hipoecoico en región cervical izquierda, así como trombosis de la vena yugular adyacente.

Se tomaron muestras mediante punción aspiración de aguja fina para citología de las adenopatías, con el resultado de "carcinoma epidermoide con rasgos sarcomatoides" y se le practicó una biopsia de la masa vaginal, compatible con carcinoma escamoso medianamente diferenciado.

El estudio analítico se completó con unos niveles elevados de antígeno asociado a células escamosas $(25 \mathrm{ng} / \mathrm{ml})$ y un patrón estrogénico acorde a la edad de la paciente.

El estudio de extensión mediante tomografía computerizada definió una masa tumoral de unos $8 \mathrm{~cm}$ de diámetro. Además de confirmarse las adenopatías cervicales, algunas de tamaño patológico, se hallaron también múltiples adenopatías retroperitoneales. Incidentalmente se describió un patrón intersticial pulmonar compatible con LAM (Figura 2). Otro hallazgo fue una imagen en riñón izquierdo compatible con angiomiolipoma (Figura 3).

\section{Discusión}

El diagnóstico de LAM, a pesar de ser anatomopatológico, se puede realizar también desde un punto de vista radiológico ${ }^{4}$, y se ha relacionado con la presencia de tumores de diferente estirpe, entre ellos los angiomioliponas ${ }^{5}$. Cabe destacar que los angiomiolipomas, si bien se consideran criterio mayor de complejo esclerosis tuberosa, suelen estar frecuentemente relacionados con $\mathrm{LAM}^{6}$ y no se vinculan necesariamente con dicha facomatosis, por lo que la presencia de estas dos entidades de manera aislada no es definitoria de la entidad. Sin embargo, la presencia de lesiones faciales hiperpigmentadas, sugerentes de angiofi-
Figura 3. TAC abdominal con imagen hipodensa en región renal izquierda, compatible con angiomiolipoma

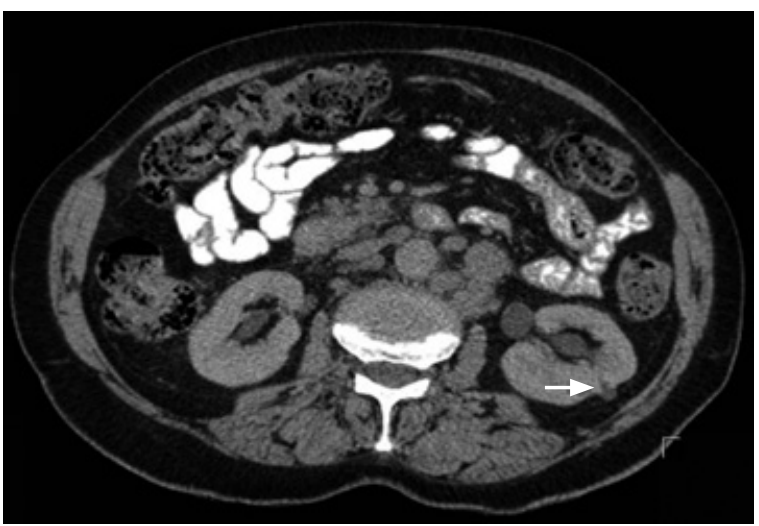

bromas, supone otro criterio mayor que permitiría clasificar el cuadro clínico como "complejo esclerosis tuberosa definida" 7 (Tabla 1).

Tabla 1. Criterios diagnósticos de Complejo Esclerosis Tuberosa
CRITERIOS MAYORES
- Angiofibroma facial
- Fibroma ungular y periungueales no traumáticos
- Parche de Shagreen (nevus de tejido conectivo)
- Máculas hipomelanóticas (más de tres)
- Tubérculo cortical
- Nódulos subependimarios
- Hamartoma retiniano
- Astrocitoma de células gigantes subependimario
- Rabdomioma cardiaco único o múltiple
- Linfangioleiomiomatosis
- Angiomiolipoma renal

\section{CRITERIOS MENORES}

- Hipoplasia del esmalte dental

- Pólipos rectales tipo hamartoma

- Quistes óseos

- Migración de líneas radiales en la sustancia blanca cerebral

- Fibromas gingivales

- Parche de retina acrómico

- Lesiones cutáneas hipomelánicas de distribución "en confeti"

- Quistes renales múltiples

\section{COMPLEJO ESCLEROSIS TUBEROSA DEFINIDO}

2 criterios mayores, o 1 mayor y 2 menores

COMPLEJO ESCLEROSIS TUBEROSA PROBABLE

- 1 mayor y 1 menor

COMPLEJO ESCLEROSIS TUBEROSA POSIBLE

1 mayor 02 más menores 
El cociente intelectual aparentemente "límite" de la paciente podría relacionarse con dicha patología, ya que, si bien no es un criterio, es frecuente la presencia de cierto retraso mental. La determinación de la mutación en los genes TSC1 o TSC2 no es necesaria para definir la enfermedad, aunque es útil para estudiar una posible agregación familiar. En este caso, no se determinaron dichas mutaciones.

Sobre la presencia de neoplasias, además de la ya comentada relación de LAM con angiomiolipomas, está descrita la asociación de LAM aislada con tumores ginecológicos, aunque sólo constan algunos casos de linfangioleiomiomatosis extrapulmonar (ganglionar) ${ }^{8}$, sin que parezca haber una relación anatomopatológica definida entre ambos. No ocurre lo mismo en la esclerosis tuberosa, donde existe asociación con varios tipos de tumores (astrocitomas de células gigantes, rabdomiomas ${ }^{9}$, hepatocarcinomas ${ }^{10}$, tumores neuroendocrinos ${ }^{11}$ ), entre los que se encuentran también los del aparato genital femenino, si bien no son muy frecuentes. Entre los descritos se encuentran: carcinoma seroso de ovario, LAM uterina, angiomiolipoma ovárico y adenocarcinoma de endometrio ${ }^{12}$. La etiopatogenia de estas neoplasias parece estar en relación con la mutación de TSC1 y TSC2 y su relación con la kinasa mTOR (blanco de la rapamicina en mamíferos), implicada en una vía de señalización relacionada con el crecimiento y la proliferación celular. La alteración de esa cascada molecular influiría en el desarrollo de tumores ginecológicos, entre los que se incluye el de cérvix ${ }^{13}$. Este planteamiento parece confirmarse con las nuevas actitudes terapéuticas, ya que se ha comprobado que el bloqueo de la vía mTOR de manera neoadyuvante con sirolimus (rapamicina) ${ }^{14}$ mejora la posterior respuesta a paclitaxel y la supervivencia de los pacientes ${ }^{15}$.

En lo que respecta a la evolución, el diagnóstico de la paciente al alta fue de carcinoma de cérvix uterino estadio IV b con metástasis linfáticas cervicales, asociado a complejo esclerosis tuberosa y LAM. Se inició tratamiento con carboplatino y paclitaxel durante 3 meses. Posteriormente la paciente presentó clínica de disfagia, por lo que se inició la administración de radioterapia en el conglomerado adenopático.

\section{Conclusión}

La asociación entre esclerosis tuberosa y neoplasias parece estar claramente relacionada y apoyada por una etiopatogenia molecular conocida. Sin embargo esta relación no está bien definida en lo referido a los tumores ginecológicos, por lo que sería necesario un estudio con mayor número de pacientes para llegar a unas adecuadas conclusiones epidemiológicas.

\section{Bibliografía}

1. Eifel P.J., Berek J.S., Markman M.A. Cancer of the cervix, vagina and vulva. En Cancer: Principles and practice of Oncology, 2008. Lippincott Williams \& Wilkins

2. Fernández Concepción 0., Gómez García A., Sardiñaz Hernández N. Esclerosis tuberosa: Revisión Rev Cubana Pediatr 1999; 71: 160-7

3. Crino P.B., M.D., Ph.D., Nathanson K.L., M.D., and Henske E.P., M.D. The Tuberous Sclerosis Complex. N Engl J Med 2006; 355:1345-56

4. Barrera E.A., Franch N.M., Vera-Sempere F., Padilla Alarcón J. Linfangioleiomiomatosis. Arch Bronconeumol. 2011; 47:85-93

5. Prieto de Paula J.M., Martín Serradilla J.I., Alonso Fernández J.I., Simal Fernández J.A. Mujer de 52 años con patrón intersticial pulmonar y retraso mental profundo. Rev Clin Esp. 2007; 207:259-61

6. Rakowski S.K., Winterkon E.B., Paul E., Steel D.J., Halpern E.F., Thiele E.A. Renal manifestations of tuberous sclerosis complex: Incidence, prognosis and predictive factors. Kidney Int. 2006; 70:1777-82.

7. Torres V., Contreras G.A., Serrano N., Páez M.C., Guzmán M.C. Complejo de la esclerosis tuberosa, revisión de tema y presentación de caso. Rev Asoc Colomb Dermatol. 2011; 19: 76-81.

8. Iwasa Y., Tachibana M., Ito H., Iwami S., Yagi H., Yamada S., et al. Extrapulmonary lymphangioleiomyomatosis in pelvic and paraaortic lymph nodes associated with uterine cancer: a report of 3 cases. Int. J Gynecol Pathol. 2011; 30: 470-5

9. Wang J.N., Yao C.T., Chen J.S., Yang Y.J., Tsai Y.C., Wu J.M. Cardiac tumors in infants and children. Acta Paediatr Taiwan. 2003; 44: 215-9

10. Yang B., Cheng W.H., Shi P.Z., Xiang J.J., Xu R.J., Liu J.H.. Coincidence of hepatocellular carcinoma and hepatic angiomyolipomas in tuberous sclerosis complex: a case report. World J. Gastroenterol. 2008 7; 14: 812-4

11. Arva N.C., Pappas J.G., Bhatla T., Raetz E.A., Macari M., Ginsburg H.B., Hajdu C.H. Well-differenciated pancreatic neuroendocrine carcinoma in tuberous sclerosis. Am J Surg Pathol. 2012; 36: 149-53

12. Jaffe J.S., Chambers J.T., Endometrial adenocarcinoma presenting in a premenopausal patient with tuberous sclerosis. J Intellect Disabil Res. 2005; 49: 463-5

13. García Galvis 0.F., Nicolae A., Cuevas Beltrán M., Alfredo García, Nogales F.F. PEComa maligno uterino asociado con complejo de esclerosis tuberosa. Patología 2008; 46:327-31

14. Bissler J.J., M.D., McCormack F.X., M.D., Young L.R., M.D., Elwing J.M., M.D., Gail Chuck, L.M.T., Jennifer M. Leonard, R.N. Sirolimus for Angiomyolipoma in Tuberous Sclerosis Complex or Lymphangioleiomyomatosis. N Engl J Med 2008; 358:140-51

15. Yi-Jen C. Targeted mTOR in Human Gynecologic Cancers. Journal of cancer molecules 2007; 3: 101-6 